\title{
Research Article Scaling Properties of a Hybrid Fermi-Ulam-Bouncer Model
}

\author{
Diego F. M. Oliveira, ${ }^{1}$ Rafael A. Bizão, ${ }^{2}$ and Edson D. Leonel ${ }^{2}$ \\ ${ }^{1}$ Departamento de Física, Instituto de Geociências e Ciências Exatas, Universidade Estadual Paulista \\ (UNESP), Avenida 24A, 1515 - Bela Vista, 13506-900 Rio Claro, SP, Brazil \\ ${ }^{2}$ Departamento de Estatística, Matemática Aplicada e Computação, Instituto de Geociências e Ciências \\ Exatas, Universidade Estadual Paulista (UNESP), Avenida 24A, 1515, Bela Vista, \\ 13506-900 Rio Claro, SP, Brazil
}

Correspondence should be addressed to Diego F. M. Oliveira, dfmo@rc.unesp.br

Received 28 January 2008; Revised 25 July 2008; Accepted 29 September 2008

Recommended by Francesco Pellicano

Some dynamical properties for a one-dimensional hybrid Fermi-Ulam-bouncer model are studied under the framework of scaling description. The model is described by using a two-dimensional nonlinear area preserving mapping. Our results show that the chaotic regime below the lowest energy invariant spanning curve is scaling invariant and the obtained critical exponents are used to find a universal plot for the second momenta of the average velocity.

Copyright (c) 2009 Diego F. M. Oliveira et al. This is an open access article distributed under the Creative Commons Attribution License, which permits unrestricted use, distribution, and reproduction in any medium, provided the original work is properly cited.

\section{Introduction}

The investigation of nonlinear dynamical systems has awaken special interest along last decades since they can explain and/or predict some phenomena until then incomprehensible. A special class of systems that present nonlinear phenomena and that can be described via recursive equations is the so-called classical nonlinear billiard problems [1, 2]. Generally, a billiard problem consists of a system in which a point-like particle moves freely inside a bounded region and suffers specular reflections with the boundaries. This system has been extensively studied during several years considering both its classical and quantized formulation.

It is well known in the literature that the phase space of billiard problems highly depends on the shape of the boundary. The dynamics of the particle might generate phase spaces of different kinds that can be settled in three different classes of universality including (i) integrable, (ii) ergodic, and (iii) mixed. A typical example of case (i) is the circle billiard, as the integrability of such a case resembles the angular momentum conservation. Two examples 
of case (ii) are the Bunimovich stadium [3] and the Sinai billiard [4]. In case (iii), the most important property present in the phase space is that chaotic seas, generally surrounding Kolmogorov-Arnold-Moser (KAM) islands, are confined by invariant spanning curves [2] sometimes also called as primary KAM tori. Particularly, such curves cross the phase plane from one side to the other one thus separating different portions of the phase space. The mixed structure in the phase portrait is such that regions of regular motion and regions with chaotic behavior could coexist together. This property is generic for nondegenerate Hamiltonian systems [5]. Some examples of systems with this particular structure are the one-dimensional Fermi-Ulam model [6-12], the bouncer model [13-19], time-dependent potentials well $[20,21]$, and many other different problems considering different degrees of freedom.

In this paper, we will consider a 1D model that is described using the formalism of discrete mappings, the so-called hybrid Fermi-Ulam-bouncer model [22, 23]. We are seeking to understand and describe a scaling property present in the chaotic sea of such a model. The model was originally proposed to merge together two different nonlinear problems commonly studied apart from each other, that is, the Fermi-Ulam model and the bouncer model. The Fermi-Ulam model (FUM) consists of a classical particle, in the total absence of any external field, which is confined to bounce between two rigid walls, where one of them is fixed and the other one is periodically time varying. The returning mechanism of the particle for a next collision with the moving wall is due to a reflection with the fixed wall. On the other hand, the bouncer model consists of a classical particle falling in a constant gravitational field and that hits a periodically oscillating wall. The returning mechanism of the bouncer model is related to the gravitational field only. Despite the very similar models, the different returning mechanisms of the two models cause profound consequences on the dynamics of the particle [24]. Particularly, depending on the combination of the control parameters and initial conditions, the average velocity of the particle reaches a constant value for sufficient long time in the FUM, while it diverges on the bouncer model. Such a divergency is basically related to the phenomenon of Fermi acceleration. Therefore, Fermi acceleration (FA) is a phenomenon in which a classical particle acquires unbounded energy from collisions with a massive moving wall $[25,26]$. Applications of FA have acquired a broad interest in different fields of physics including plasma physics [27], astrophysics [28, 29], atomic physics [30], optics [31, 32], and even in the well-known time-dependent billiard problems [33, 34]. The interesting posed question that should be answered is whether the FA results from the nonlinear dynamics itself considering the complete absence of any imposed random motion. An one-dimensional model exhibiting such a phenomenon, which is modelled by a nonlinear mapping, is the bouncer model. For two-dimensional time-dependent billiards (billiards with moving boundaries), the answer for this question is not unique and it depends on the kind of phase space for the corresponding static version of the problem. Therefore, as conjectured by Loskutov et al. [35] (such a conjecture is known in the literature as the LRA conjecture), the regular dynamics for a fixed boundary implies a bound to the energy gained by the bouncing particle, but the chaotic dynamics of a billiard with a fixed boundary is a sufficient condition for FA in the system when a boundary perturbation is introduced. Such a conjecture was confirmed in different billiards [36-38]. It was, therefore, observed recently FA in a time varying elliptical billiard [39]. The oval billiard, however, seems not to exhibit FA for the breathing case [40].

Thus, the hybrid Fermi-Ulam-bouncer model consists of a classical particle which is confined in and bouncing elastically between two rigid walls in the presence of a constant gravitational field. Thus, properties that are individually observed in the FUM and bouncer 
model come together and coalesce in the hybrid Fermi-Ulam-bouncer model [22]. As we will show, the phase space of this model is of mixed kind. Our main goal is to characterize some properties on the chaotic regime for the region below the first invariant spanning curve, particularly the behavior of the average velocity and the deviation of the average velocity.

This paper is organized as follows. In Section 2, we give all the details needed for the construction of the nonlinear mapping. We illustrate some results for the phase space of the model considering both the simplified and the complete versions of the Fermi-Ulam-bouncer model. The scaling hypotheses are present and numerical results are discussed in Section 3. Finally, in Section 4, we draw our conclusions.

\section{The Model and the Mapping}

We discuss in this section all the details needed for the mapping construction. We also present the phase space and obtain the positive Lyapunov exponent for the low-energy chaotic sea. The one-dimensional hybrid Fermi-Ulam-bouncer model thus consists of a classical particle confined to bounce elastically between two rigid walls. One of the walls is assumed to be fixed at the position $y=l$, while the other one moves periodically in time according to the equation $y_{w}(t)=\varepsilon \cos (w t)$. Here, $\varepsilon$ and $w$ denote, respectively, the amplitude of oscillation and the frequency of the moving wall. Additionally, the particle is suffering the action of a constant gravitational field $g^{\prime}$. As it is so usual in the literature, the dynamics of the particle is described in terms of a two-dimensional nonlinear area preserving map $T$ that gives the velocity of the particle $v_{n}$ and the corresponding time $t_{n}$ at the instant of the $n$th impact with the moving wall, that is, $T\left(v_{n}, t_{n}\right)=\left(v_{n+1}, t_{n+1}\right)$. Before we write the equations of the mapping, it is convenient to define dimensionless and much more appropriated variables to describe the dynamics of the particle. We define $\epsilon=\varepsilon / l, g=g^{\prime} /\left(w^{2} l\right)$, and $V_{n}=v_{n} /(w l)$. Finally, we measure the time in terms of $\phi_{n}=w t_{n}$. Incorporating this new set of variables in the dynamics, the map is written as

$$
T:\left\{\begin{array}{l}
V_{n+1}=V_{n}^{*}+g \phi_{c}-2 \epsilon \sin \left(\phi_{n+1}\right), \\
\phi_{n+1}=\left(\phi_{n}+\Delta T_{n}\right) \bmod (2 \pi),
\end{array}\right.
$$

where the expressions for both $V_{n}^{*}$ and $\Delta T_{n}$ depend on the kind of collision occurs. There are three different possible situations, namely (i) multiple collisions, (ii) collisions without reflection in the upper wall, and (iii) collisions with reflection in the upper wall. Considering the first case, where the particle suffers an impact before leaving the collision zone, which is given by the interval $y \in[-\epsilon, \epsilon]$, the expressions are $V_{n}^{*}=-V_{n}$ and $\Delta T_{n}=\phi_{c}$, where $\phi_{c}$ is obtained as the smallest solution of $G\left(\phi_{c}\right)=0$ with $\phi_{c} \in(0,2 \pi]$. Such solution is equivalent to the position of the particle being the same as the position of the moving wall. Thus, the transcendental equation $G\left(\phi_{c}\right)$ is given by

$$
G\left(\phi_{c}\right)=\epsilon \cos \left(\phi_{n}+\phi_{c}\right)-\epsilon \cos \left(\phi_{n}\right)-V_{n} \phi_{c}+\frac{g \phi_{c}^{2}}{2} .
$$

If $G\left(\phi_{c}\right)$ does not have a solution in the interval $\phi_{c} \in(0,2 \pi]$, the particle leaves the collision zone without suffering a successive hit. Considering the case (ii), the needed condition for the particle not colliding with the upper wall after leaving the collision zone is 
$V_{n} \leq \sqrt{2 g\left[1-\epsilon \cos \left(\phi_{n}\right)\right]}$. In this case, $V_{n}^{*}=\sqrt{V_{n}^{2}+2 g \epsilon\left[\cos \left(\phi_{n}\right)-1\right]}$ and $\Delta T_{n}=\phi_{u}+\phi_{d}+\phi_{c}$, where the auxiliary terms assume the following expressions:

$$
\begin{aligned}
\phi_{u} & =\frac{V_{n}}{g}, \\
\phi_{d} & =\frac{\sqrt{V_{n}^{2}+2 g \epsilon\left[\cos \left(\phi_{n}\right)-1\right]}}{g} .
\end{aligned}
$$

The term $\phi_{c}$ is obtained by solution of $F\left(\phi_{c}\right)=0$. Again, considering the condition that matches the same position of the moving wall and of the particle. Thus, $F\left(\phi_{c}\right)$ is given by

$$
F\left(\phi_{c}\right)=\epsilon \cos \left(\phi_{n}+\phi_{u}+\phi_{d}+\phi_{c}\right)-\epsilon-V_{n}^{*} \phi_{c}+\frac{g \phi_{c}^{2}}{2}
$$

Finally, for the case (iii), where $V_{n}>\sqrt{2 g\left[1-\epsilon \cos \left(\phi_{n}\right)\right]}$, the corresponding expressions for $V_{n}^{*}$ and $\Delta T_{n}$ are the same as those of the case (ii). However, the auxiliary terms, $\phi_{u}$ and $\phi_{d}$, assume the following expressions:

$$
\begin{aligned}
\phi_{u} & =\frac{V_{n}-\sqrt{V_{n}^{2}-2 g\left[1-\epsilon \cos \left(\phi_{n}\right)\right]}}{g}, \\
\phi_{d} & =\frac{\sqrt{V_{n}^{2}+2 g \epsilon\left[\epsilon \cos \left(\phi_{n}\right)-1\right]}-\sqrt{V_{n}^{2}-2 g\left[1-\epsilon \cos \left(\phi_{n}\right)\right]}}{g} .
\end{aligned}
$$

The value of $\phi_{c}$ is obtained from (2.4) using, however, the new expressions for $\phi_{u}$ and $\phi_{d}$.

Figure 1(a) shows the phase space for the complete hybrid Fermi-Ulam-bouncer model generated from (2.1). The control parameters used in Figure 1 are $\epsilon=10^{-3}$ and $g=$ $5.628 \times 10^{-5}$. It is easy to see the presence of KAM islands surrounded by a chaotic sea, that is, limited by an invariant spanning curve. The presence of the invariant spanning curves in the phase space implies that the particle cannot acquire unlimited energy. To illustrate the behavior of the average energy, we define

$$
E_{j}=\frac{1}{n+1} \sum_{i=0}^{n}{\overline{V^{2}}}_{i, j}
$$

and finally obtain the average energy as

$$
\bar{E}(n, \epsilon)=\frac{1}{M} \sum_{j=1}^{M} E_{j},
$$

where $M$ corresponds to an ensemble of different initial conditions. 


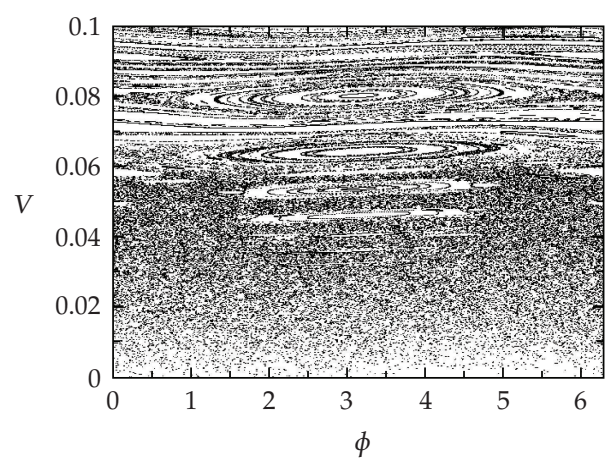

(a)

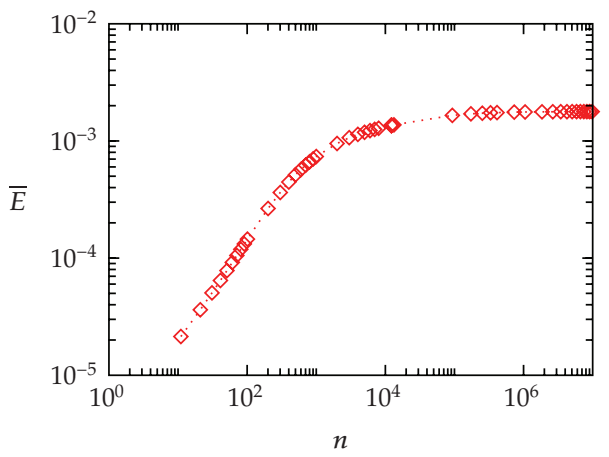

(b)

Figure 1: (a) Phase space for the complete hybrid Fermi-Ulam-bouncer model. (b) Behavior of the average energy, $\bar{E}$, as function of $n$. The control parameters used in (a) and (b) were $\epsilon=10^{-3}$ and $g=5.628 \times 10^{-5}$.

Figure 1(b) shows the behavior of the average energy, $\bar{E}$, as a function of $n$. We can see that the energy grows for small $n$ and then, after a changeover, it reaches a regime of saturation for large $n$. The initial velocity was considered fixed as $V_{0}=2.1 \epsilon$, while we have considered an ensemble of $M=5000$ different initial phases $\phi_{0}$ uniformly distributed in the interval $[0,2 \pi)$. Each initial condition was evolved up to $10^{7}$ iterations. Since the instant of each impact of the particle with the moving wall can only be obtained numerically, the computational time for evaluation of (2.7) is very large. As an attempt to reduce the time consuming in the numerical simulations, we will discuss in Section 2.1 a simplified version of the hybrid Fermi-Ulam-bouncer model.

\subsection{A Simplified Hybrid Fermi-Ulam-Bouncer Model}

In this section, we discuss a simplification used in the model. For the complete model, the instant of the impact of the particle with the moving wall is obtained via a solution of a transcendental equation, which yields the simulations to be long-time consuming. However, instead of considering solving transcendental equations, we will use a simplification in the model which is commonly used in the literature [2]. We will suppose that both walls are fixed (one of them is fixed at $y=0$ and the other one is fixed at $y=l$ ), but that, when the particle suffers a collision with one of then (say, with the one at $y=0$ ), the particle exchanges momentum and energy as if the wall was moving. This simplification carries a great advantage of allowing us to speed up our numerical simulations when compared to the complete version of the model. Such a simplification also retains the nonlinearity of the problem. Considering the same dimensionless variables defined for the complete model, the map for the simplified hybrid Fermi-Ulam-bouncer model can be written as

$$
T:\left\{\begin{array}{l}
V_{n+1}=\left|V_{n}-2 \epsilon \sin \left(\phi_{n+1}\right)\right|, \\
\phi_{n+1}=\left(\phi_{n}+\Delta T_{n}\right) \bmod (2 \pi) .
\end{array}\right.
$$


The modulus function used in the equation of the velocity on the mapping (2.8) was introduced to preserve the particle into the region between the walls. The expressions for $\Delta T_{n}$ depend on the following conditions:

(1) collision without reflection in the upper wall. Such a condition is verified if $V_{n} \leq \sqrt{2 g\left(1-\epsilon \cos \left(\phi_{n}\right)\right)}$. In this case, the particle rises and decelerates due to the gravitational field only until reaches instantaneously the rest. Then the particle is accelerated downward until it collides with the wall at $y=0$. Thus, $\Delta T_{n}$ is given by

$$
\Delta T_{n}=\frac{2 V_{n}}{g}
$$

(2) Collision with reflection in the upper wall. For such a collision, the condition that must be observed is $V_{n}>\sqrt{2 g\left(1-\epsilon \cos \left(\phi_{n}\right)\right)}$. In this case, the particle goes in the upward direction and hits the upper wall. It is then reflected downward and is also accelerated by the gravitational field. The expression for $\Delta T_{n}$ is written as

$$
\Delta T_{n}=\frac{V_{n}-\sqrt{V_{n}^{2}-2 g}}{g} .
$$

The mapping (2.8) preserves the phase space measure since that $\operatorname{det}(J)= \pm 1$.

The phase space for the mapping (2.8) is shown in Figure 2(a) for the same control parameters used in Figure 1. We have also evaluated numerically the positive Lyapunov exponent for the chaotic sea of Figure 2(a). It is well known that the Lyapunov exponent has great applicability as a practical tool that can quantify the average expansion or contraction rate for a small volume of initial conditions. As discussed in [41], the Lyapunov exponents are defined as

$$
\lambda_{j}=\lim _{n \rightarrow \infty} \frac{1}{n} \ln \left|\Lambda_{j}\right|, \quad j=1,2,
$$

where $\Lambda_{j}$ are the eigenvalues of $M=\prod_{i=1}^{n} J_{i}\left(V_{i}, \phi_{i}\right)$ and $J_{i}$ is the Jacobian matrix evaluated over the orbit $\left(V_{i}, \phi_{i}\right)$. However, a direct implementation of a computational algorithm to evaluate (2.11) has a severe limitation to obtain $M$. Even in the limit of short $n$, the components of $M$ can assume very different orders of magnitude for chaotic orbits and periodic attractors, yielding impracticable the implementation of the algorithm. In order to avoid such problem, we note that $J$ can be written as $J=\Theta T$, where $\Theta$ is an orthogonal matrix and $T$ is a right triangular matrix. Thus, we rewrite $M$ as $M=J_{n} J_{n-1}, \ldots, J_{2} \Theta_{1} \Theta_{1}^{-1} J_{1}$, where $T_{1}=\Theta_{1}^{-1} J_{1}$. A product of $J_{2} \Theta_{1}$ defines a new $J_{2}^{\prime}$. In a next step, it is easy to show that $M=J_{n} J_{n-1}, \ldots, J_{3} \Theta_{2} \Theta_{2}^{-1} J_{2}^{\prime} T_{1}$. The same procedure can be used to obtain $T_{2}=\Theta_{2}^{-1} J_{2}^{\prime}$, and so on. Using this procedure, the problem is reduced to evaluate the diagonal elements of $T_{i}$ : $T_{11}^{i}, T_{22}^{i}$. Finally, the Lyapunov exponents are now given by

$$
\lambda_{j}=\lim _{n \rightarrow \infty} \frac{1}{n} \sum_{i=1}^{n} \ln \left|T_{j j}^{i}\right|, \quad j=1,2 .
$$




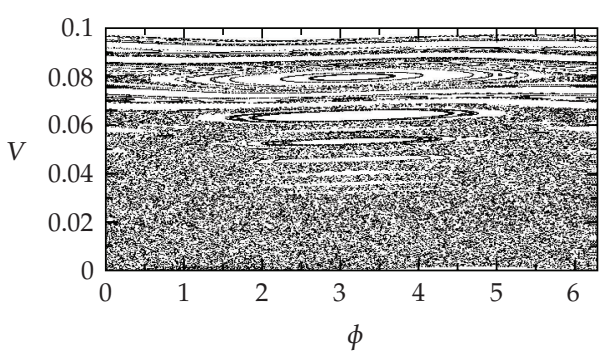

(a)

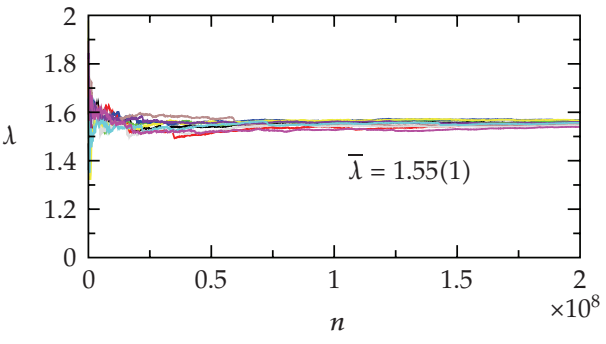

(b)

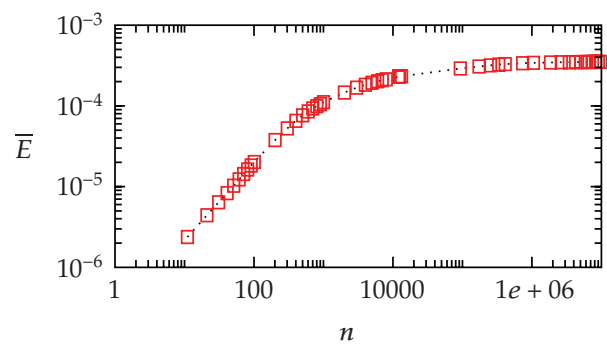

(c)

Figure 2: (a) Phase space generated from iteration of mapping (2.8) for the control parameters $\epsilon=10^{-3}$ and $g=5.628 \times 10^{-5}$. (b) Behavior of the positive Lyapunov exponent of the chaotic sea for the same control parameters used in (a). The average value obtained was $\bar{\lambda}=1.55 \pm 0.01$, where the error 0.01 corresponds to the standard deviation of the 10 samples. (c) Behavior of the average energy, $\bar{E}$, as function of $n$.

If at least one of the $\lambda_{j}$ is positive, then the orbit is classified as chaotic. Figure 2(b) shows the behavior of the positive Lyapunov exponent plotted against the number of collisions $n$ with the wall located at $y=0$ for 10 different initial conditions on the chaotic sea. The control parameters used in the construction of Figure 2 were $\epsilon=10^{-3}$ and $g=5.628 \times 10^{-5}$. The average of the positive Lyapunov exponent for the ensemble of the 10-time series gives $\bar{\lambda}=1.55 \pm 0.01$, where the value 0.01 corresponds to the standard deviation of the ten samples. Finally, in Figure 2(c), we present the behavior of the average energy of the particle for the simplified model.

\section{Scaling Analysis}

The main goal of this section is to describe a scaling present in the low-energy regime. We discuss in full detail the investigation for the simplified and then, at the end of section, we present the corresponding results for the complete version. Thus, we now discuss the procedures used to obtain the average velocity on the chaotic low-energy region. The average velocity (average along an orbit) is defined as

$$
\bar{V}(n, \epsilon)=\frac{1}{n+1} \sum_{i=0}^{n} V_{i}
$$


Since we obtain the average velocity, it is also easy to obtain the deviation of the average velocity, sometimes also called as the second momenta of $\bar{V}$. It is defined as

$$
\omega(n, \epsilon)=\frac{1}{M} \sum_{i=1}^{M} \sqrt{\overline{V_{i}^{2}}(n, \epsilon)-\bar{V}_{i}^{2}(n, \epsilon)}
$$

where the sum on $M$ refers to an average over an ensemble of $M$ different initial conditions. In order to iterate (3.2) for the simplified version of the model, we evolved our simulations considering an ensemble of $M=5 \times 10^{3}$ different initial conditions. Figure 3 shows the behavior of the deviation around the average velocity for the simplified model as function of $n$ for three different control parameters, as labelled in the figures. It is easy to see in Figure 3 two different kinds of behaviors. For short $n$, the deviation of the average velocity grows according to a power law and suddenly it bends toward a regime of saturation for long enough values of $n$. The changeover from growth to the saturation is marked by a cross-over iteration number $n_{x}$. It must be emphasized that different values of the parameter $\epsilon$ generate different behaviors for short $n$. However, applying the transformation $n \rightarrow n \epsilon^{2}$ coalesces all the curves at short $n$, as shown in Figure 3(b). We can see that different values of $\epsilon$ yield each curve to saturate at distinct values, thus we can suppose that

(1) when $n \ll n_{x}$, the deviation of the average velocity grows according to

$$
\omega\left(n \epsilon^{2}, \epsilon\right) \propto\left(n \epsilon^{2}\right)^{\beta}
$$

where the exponent $\beta$ is a critical exponent;

(2) as the iteration number increases, $n \gg n_{x}$, the deviation of the average velocity approaches a regime of saturation, that is described as

$$
\omega_{\text {sat }}(\epsilon) \propto \epsilon^{\alpha},
$$

where the exponent $\alpha$ is also a critical exponent;

(3) the cross-over iteration number that marks the change from growth to the saturation is written as

$$
n_{x} \propto \epsilon^{z}
$$

where $z$ is a dynamic exponent.

After considering these three initial suppositions, we are now able to describe the deviation of the average velocity in terms of a scaling function of the type

$$
\omega\left(n \epsilon^{2}, \epsilon\right)=l \omega\left(l^{a} n \epsilon^{2}, l^{b} \epsilon\right),
$$

where $l$ is the scaling factor, $a$ and $b$ are scaling exponents. If we chose properly the scaling factor $l$, we can relate the scaling exponents $a$ and $b$ with the critical exponents $\alpha, \beta$, and $z$. 


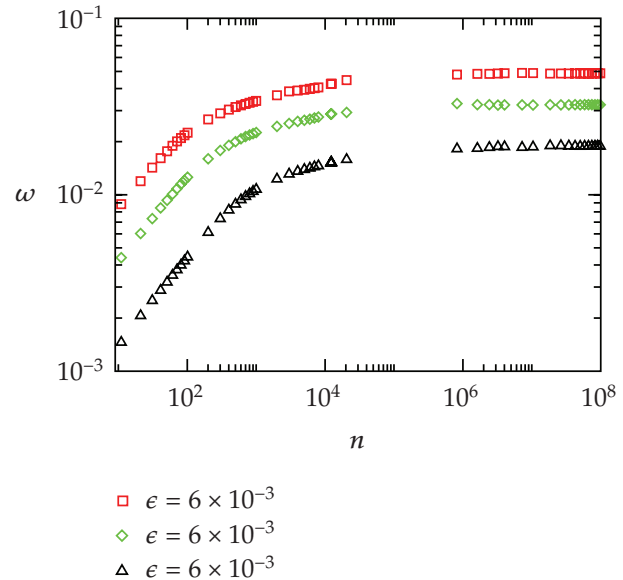

(a)

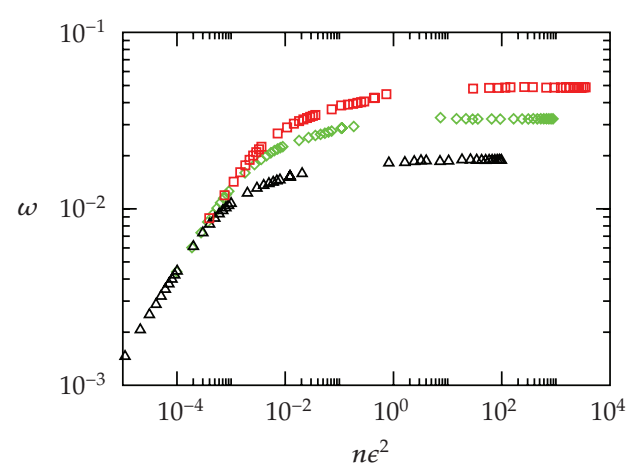

ㅁ $\epsilon=6 \times 10^{-3}$

$\diamond \epsilon=6 \times 10^{-3}$

$\Delta \epsilon=6 \times 10^{-3}$

Figure 3: (a) Behavior of the deviation of the average velocity for different values of the control parameter $\epsilon$ for the simplified model. (b) Their initial collapse after the transformation $n \epsilon^{2}$ for the simplified model. The control parameter $g$ was fixed as $g=5.628 \times 10^{-5}$.

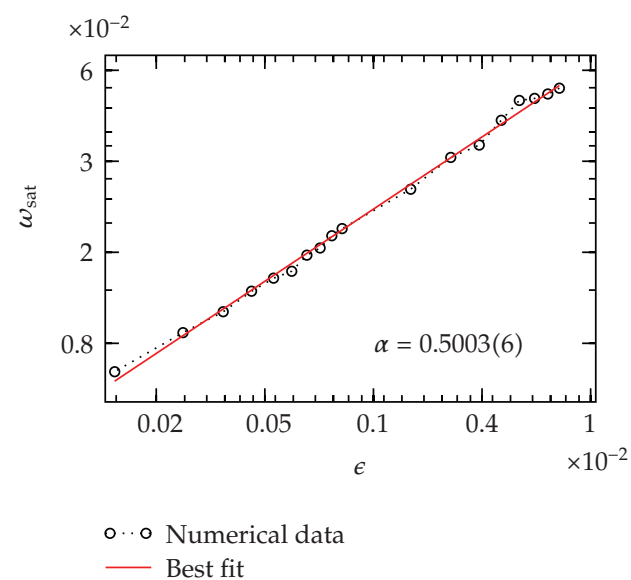

(a)

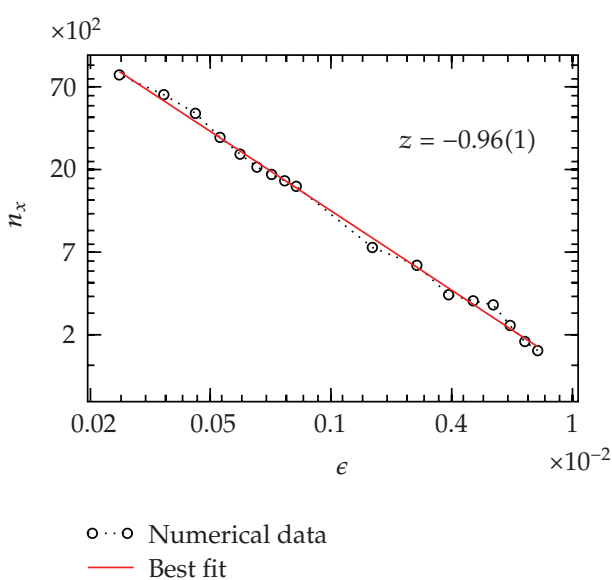

(b)

Figure 4: (a) Plot of $\omega_{\text {sat }}$ as function of the control parameter $\epsilon$ for the simplified model. (b) Behavior of the cross-over number $n_{x}$ against $\epsilon$ for the simplified model.

We begin considering that $l=\left(n \epsilon^{2}\right)^{-1 / a}$. Thus, (3.6) is rewritten as

$$
\omega\left(n \epsilon^{2}, \epsilon\right)=\left(n \epsilon^{2}\right)^{-1 / a} \omega_{1}\left(\left(n \epsilon^{2}\right)^{-b / a} \epsilon\right),
$$

where the function $\omega_{1}\left(\left(n \epsilon^{2}\right)^{-b / a} \epsilon\right)=\omega\left(1,\left(n \epsilon^{2}\right)^{-b / a} \epsilon\right)$ is assumed to be constant for $n \ll n_{x}$. Comparing (3.3) and (3.7), we obtain $\beta=-1 / a$.

Choosing now $l^{b} \epsilon=1$, we have $l=\epsilon^{-1 / b}$ and (3.6) is given by

$$
\omega\left(n \epsilon^{2}, \epsilon\right)=\epsilon^{-1 / b} \omega\left(\epsilon^{-a / b} n \epsilon^{2}\right),
$$




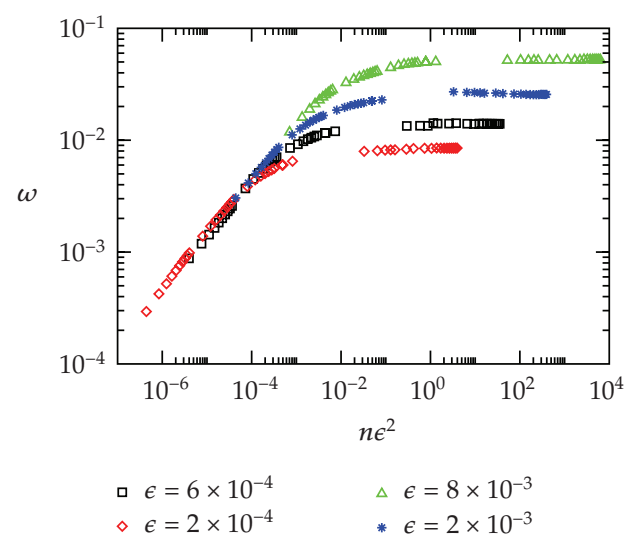

(a)

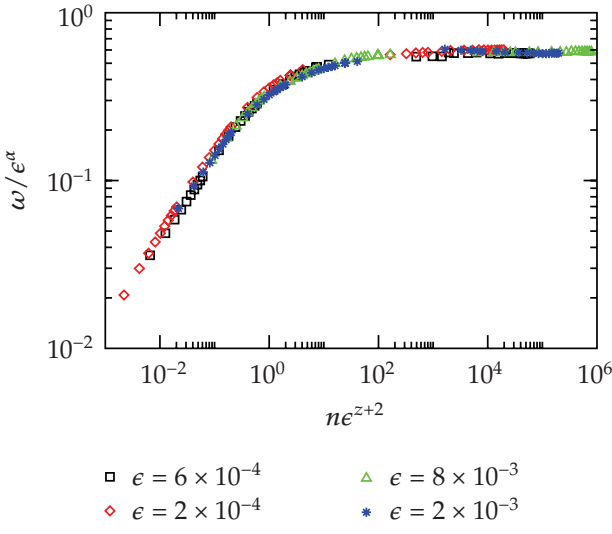

(b)

Figure 5: (a) Different curves of the $\omega$ for four different control parameters for the simplified model. (b) Their collapse onto a single and universal plots.

where the function $\omega_{2}$ is defined as $\omega_{2}\left(\epsilon^{-a / b} n \epsilon^{2}\right)=\omega\left(\epsilon^{-a / b} n \epsilon^{2}, 1\right)$. It is also assumed as constant for $n \gg n_{x}$. An immediate comparison of (3.4) and (3.6) gives us $\alpha=-1 / b$. Given the two different expressions of the scaling factor $l$, we obtain a relation for the dynamic exponent $z$, that is, given by

$$
z=\frac{\alpha}{\beta}-2
$$

Note that the scaling exponents are determined if the critical exponents $\beta$ and $\alpha$ were numerically obtained. The exponent $\beta$ is obtained from a power law fitting for the deviation of the average velocity curves for the parameter $\epsilon \in\left[10^{-4}, 10^{-2}\right]$ for short iteration number. Thus, the average of these values gives that $\beta=0.502(4) \cong 1 / 2$. Figure 4 shows the behaviors of (a) $\omega_{\text {sat }} \times \epsilon$ and (b) $n_{x} \times \epsilon$. Applying power law fittings on the figure, we obtain $\alpha=0.5003(6) \cong 0.5$ and $z=-0.96(1) \cong-1$. We can also obtain the exponent $z$ considering (3.9) and the previous values of both $\alpha$ and $\beta$; we found that $z=-1.003(6) \cong-1$. Such result indeed agrees with our numerical result. In order to confirm the initial hypotheses, and since the values of the scaling exponents $\alpha, \beta$, and $z$ are now known, we will collapse all the curves onto a single and universal plots, as demonstrated in Figure 5. With this good collapse of all the curves of the deviation of the average velocity and considering that the critical exponents are $\alpha \cong 0.5$, $\beta \cong 0.5$ and $z \cong-1$, we can conclude that the hybrid Fermi-Ulam-bouncer model belongs to the same class of universality of a periodically corrugated waveguide [42]. It also belongs to the same class of universality of the Fermi-Ulam $[43,44]$ itself and that the presence of a gravitational field does not seem to create a new universality class, at least for the control parameters considered in the present study.

Let us now discuss our numerical results for the complete version of the model. Once the equations of the mapping now be solved numerically, we have considered an ensemble of less different initial conditions. Such a consideration is mainly to produce a simulation not so longer. However, it is still relevant to characterize statistical properties of the model. For the complete version of the model, we have considered an ensemble of $M=10^{3}$ different initial conditions. The behavior of $\omega$ for the complete version is rather similar to that observed for 


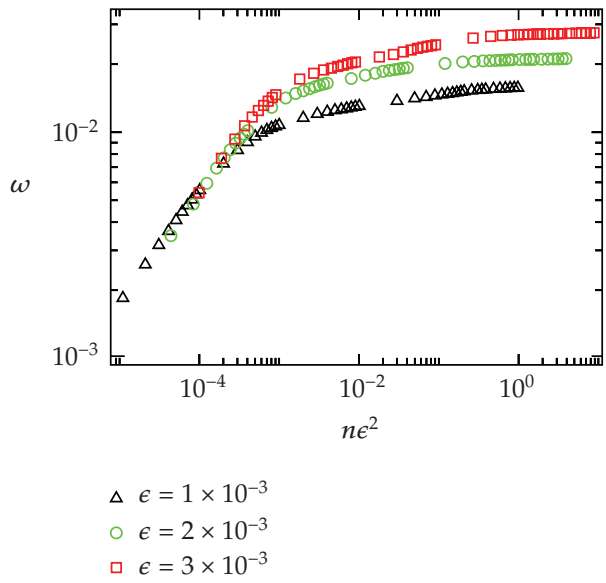

(a)

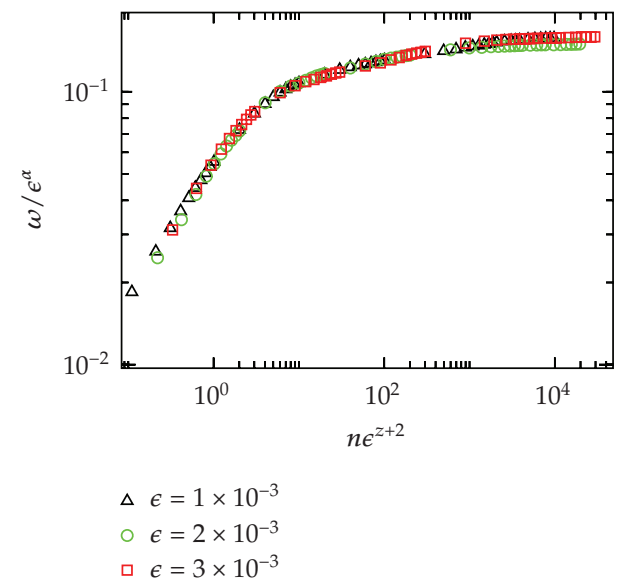

(b)

Figure 6: (a) Different curves of the $\omega$ for three different control parameters for the complete model. (b) Their collapse onto a single and universal plots.

the simplified version of the model. After an extensive simulation, we obtain that the critical exponents are $\beta=0.50(2) \cong 0.5, \alpha=0.501(3)$, and $z=-0.95$ (3). Evaluating (3.9), we found that $z=-1.00$ (3). These critical exponents also allow us to produce a good collapse of all the curves of $\omega$ onto a single and universal plots, as shown in Figure 6. Thus, we can conclude that the scaling properties are unaffected by considering the simplified or complete versions of the model.

\section{Final Remarks}

As a final remark of the present paper, we have studied a simplified and the complete version of the hybrid Fermi-Ulam-bouncer model considering elastic collisions with the walls. We show that the average energy as well as the deviation around the average velocity for chaotic orbits for both the complete and simplified versions of the model exhibit scaling properties with the same critical exponents. Moreover, we have shown that there is an analytical relation between the critical exponents $\alpha, \beta$, and $z$. Our scaling hypotheses are confirmed by a good collapse of all the curves of $\omega$ onto a single and universal plot, therefore, confirming that this model also belongs to the same class of universality of the Fermi-Ulam model [43], for the range of control parameters studied, and the periodically corrugated waveguide [42].

\section{Acknowledgment}

D. F. M. Oliveira and R. A. Bizão are grateful to CNPq; E. D. Leonel thanks FAPESP, FUNDUNESP, and CNPq for financial support.

\section{References}

[1] M. V. Berry, "Regularity and chaos in classical mechanics, illustrated by three deformations of a circular 'billiard'," European Journal of Physics, vol. 2, no. 2, pp. 91-102, 1981. 
[2] A. J. Lichtenberg and M. A. Lieberman, Regular and Chaotic Dynamics, vol. 38 of Applied Mathematical Sciences, Springer, New York, NY, USA, 2nd edition, 1992.

[3] L. A. Bunimovich, "On the ergodic properties of nowhere dispersing billiards," Communications in Mathematical Physics, vol. 65, no. 3, pp. 295-312, 1979.

[4] Y. G. Sinai, "Dynamical systems with elastic reflections," Russian Mathematical Surveys, vol. 25, no. 2, pp. 137-189, 1970.

[5] A. M. Ozorio de Almeida, Hamiltonian Systems: Chaos and Quantization, Cambridge Monographs on Mathematical Physics, Cambridge University Press, Cambridge, UK, 1988.

[6] D. G. Ladeira and J. K. L. da Silva, “Time-dependent properties of a simplified Fermi-Ulam accelerator model," Physical Review E, vol. 73, no. 2, Article ID 026201, 6 pages, 2006.

[7] E. D. Leonel, J. K. L. da Silva, and S. O. Kamphorst, "On the dynamical properties of a Fermi accelerator model," Physica A, vol. 331, no. 3-4, pp. 435-447, 2004.

[8] A. K. Karlis, P. K. Papachristou, F. K. Diakonos, V. Constantoudis, and P. Schmelcher, "Hyperacceleration in a stochastic Fermi-Ulam model," Physical Review Letters, vol. 97, no. 19, Article ID 194102, 4 pages, 2006.

[9] A. K. Karlis, P. K. Papachristou, F. K. Diakonos, V. Constantoudis, and P. Schmelcher, "Fermi acceleration in the randomized driven Lorentz gas and the Fermi-Ulam model," Physical Review E, vol. 76, no. 1, Article ID 016214, 17 pages, 2007.

[10] J. V. José and R. Cordery, "Study of a quantum Fermi-acceleration model," Physical Review Letters, vol. 56, no. 4, pp. 290-293, 1986.

[11] E. D. Leonel, D. F. M. Oliveira, and R. E. de Carvalho, "Scaling properties of the regular dynamics for a dissipative bouncing ball model," Physica A, vol. 386, no. 1, pp. 73-78, 2007.

[12] D. F. M. Oliveira and E. D. Leonel, "The Feigenbaum's $\delta$ for a high dissipative bouncing ball model," Brazilian Journal of Physics, vol. 38, no. 1, pp. 62-64, 2008.

[13] R. M. Everson, "Chaotic dynamics of a bouncing ball," Physica D, vol. 19, no. 3, pp. 355-383, 1986.

[14] P. J. Holmes, "The dynamics of repeated impacts with a sinusoidally vibrating table," Journal of Sound and Vibration, vol. 84, no. 2, pp. 173-189, 1982.

[15] G. A. Luna-Acosta, "Regular and chaotic dynamics of the damped Fermi accelerator," Physical Review A, vol. 42, no. 12, pp. 7155-7162, 1990.

[16] T. L. Vincent and A. I. Mees, "Controlling a bouncing ball," International Journal of Bifurcation and Chaos in Applied Sciences and Engineering, vol. 10, no. 3, pp. 579-592, 2000.

[17] E. D. Leonel and A. L. P. Livorati, "Describing Fermi acceleration with a scaling approach: the Bouncer model revisited," Physica A, vol. 387, no. 5-6, pp. 1155-1160, 2008.

[18] A. C. J. Luo and R. P. S. Han, "The dynamics of a bouncing ball with a sinusoidally vibrating table revisited," Nonlinear Dynamics, vol. 10, no. 1, pp. 1-18, 1996.

[19] A. C. J. Luo, "An unsymmetrical motion in a horizontal impact oscillator," Journal of Vibration and Acoustics, vol. 124, no. 3, pp. 420-426, 2002.

[20] E. D. Leonel and P. V. E. McClintock, "Scaling properties for a classical particle in a time-dependent potential well," Chaos, vol. 15, no. 3, Article ID 033701, 7 pages, 2005.

[21] J. L. Mateos, "Traversal-time distribution for a classical time-modulated barrier," Physics Letters A, vol. 256, no. 2-3, pp. 113-121, 1999.

[22] E. D. Leonel and P. V. E. McClintock, "A hybrid Fermi-Ulam-bouncer model," Journal of Physics A, vol. 38, no. 4, pp. 823-839, 2005.

[23] D. G. Ladeira and E. D. Leonel, “Dynamical properties of a dissipative hybrid Fermi-Ulam-bouncer model," Chaos, vol. 17, no. 1, Article ID 013119, 7 pages, 2007.

[24] A. J. Lichtenberg, M. A. Lieberman, and R. H. Cohen, "Fermi acceleration revisited," Physica D, vol. 1, no. 3, pp. 291-305, 1980.

[25] S. M. Ulam, "On some statistical properties of dynamical systems," in Proceedings of the 4th Berkeley Symposium on Mathematical Statistics and Probability, vol. 3, pp. 315-320, University of California Press, Berkeley, Calif, USA, June-July 1960.

[26] J. M. Hammerseley, "On the dynamical disequilibrium of individual particles," in Proceedings of the 4th Berkeley Symposium on Mathematical Statistics and Probability, vol. 3, p. 79, University of California Press, Berkeley, Calif, USA, June-July 1960.

[27] A. V. Milovanov and L. M. Zelenyi, "“Strange” Fermi processes and power-law nonthermal tails from a self-consistent fractional kinetic equation," Physical Review E, vol. 64, no. 5, Article ID 052101, 4 pages, 2001. 
[28] A. Veltri and V. Carbone, "Radiative intermittent events during Fermi's stochastic acceleration," Physical Review Letters, vol. 92, no. 14, Article ID 143901, 4 pages, 2004.

[29] K. Kobayakawa, Y. S. Honda, and T. Samura, "Acceleration by oblique shocks at supernova remnants and cosmic ray spectra around the knee region," Physical Review D, vol. 66, no. 8, Article ID 083004, 11 pages, 2002.

[30] G. Lanzanò, E. De Filippo, D. Mahboub, et al., "Fast electron production at intermediate energies: evidence for Fermi shuttle acceleration and for deviations from simple relativistic kinematics," Physical Review Letters, vol. 83, no. 22, pp. 4518-4521, 1999.

[31] G. L. Gutsev, P. Jena, and R. J. Bartlett, "Two thermodynamically stable states in $\mathrm{SiO}^{-}$and $\mathrm{PN}^{-}$," Physical Review A, vol. 58, no. 6, pp. 4972-4974, 1998.

[32] A. Steane, P. Szriftgiser, P. Desbiolles, and J. Dalibard, "Phase modulation of atomic de Broglie waves," Physical Review Letters, vol. 74, no. 25, pp. 4972-4975, 1995.

[33] A. Loskutov and A. B. Ryabov, "Particle dynamics in time-dependent stadium-like billiards," Journal of Statistical Physics, vol. 108, no. 5-6, pp. 995-1014, 2002.

[34] S. O. Kamphorst and S. P. de Carvalho, "Bounded gain of energy on the breathing circle billiard," Nonlinearity, vol. 12, no. 5, pp. 1363-1371, 1999.

[35] A. Loskutov, A. B. Ryabov, and L. G. Akinshin, "Properties of some chaotic billiards with timedependent boundaries," Journal of Physics A, vol. 33, no. 44, pp. 7973-7986, 2000.

[36] R. E. de Carvalho, F. C. Souza, and E. D. Leonel, "Fermi acceleration on the annular billiard," Physical Review E, vol. 73, no. 6, Article ID 066229, 10 pages, 2006.

[37] A. Yu. Loskutov, A. B. Ryabov, and L. G. Akinshin, "Mechanism of Fermi acceleration in dispersing billiards with time-dependent boundaries," Journal of Experimental and Theoretical Physics, vol. 89, no. 5, pp. 966-974, 1999.

[38] R. E. de Carvalho, F. C. de Souza, and E. D. Leonel, "Fermi acceleration on the annular billiard: a simplified version," Journal of Physics A, vol. 39, no. 14, pp. 3561-3573, 2006.

[39] F. Lenz, F. K. Diakonos, and P. Schmelcher, "Tunable Fermi acceleration in the driven elliptical billiard," Physical Review Letters, vol. 100, no. 1, Article ID 014103, 4 pages, 2008.

[40] S. O. Kamphorst, E. D. Leonel, and J. K. L. da Silva, "The presence and lack of Fermi acceleration in nonintegrable billiards," Journal of Physics A, vol. 40, no. 37, pp. F887-F893, 2007.

[41] J.-P. Eckmann and D. Ruelle, "Ergodic theory of chaos and strange attractors," Reviews of Modern Physics, vol. 57, no. 3, pp. 617-656, 1985.

[42] E. D. Leonel, “Corrugated waveguide under scaling investigation," Physical Review Letters, vol. 98 , no. 11, Article ID 114102, 4 pages, 2007.

[43] E. D. Leonel, P. V. E. McClintock, and J. K. L. da Silva, “Fermi-Ulam accelerator model under scaling analysis," Physical Review Letters, vol. 93, no. 1, Article ID 014101, 4 pages, 2004.

[44] J. K. L. da Silva, D. G. Ladeira, E. D. Leonel, P. V. E. McClintock, and S. O. Kamphorst, "Scaling properties of the Fermi-Ulam accelerator model," Brazilian Journal of Physics, vol. 36, no. 3A, pp. 700707, 2006. 


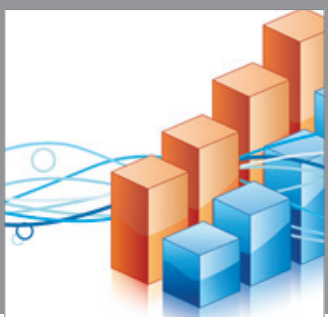

Advances in

Operations Research

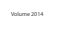

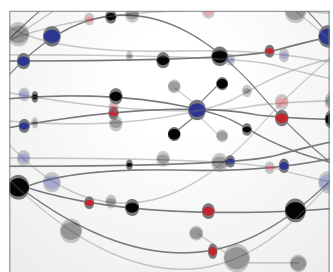

\section{The Scientific} World Journal
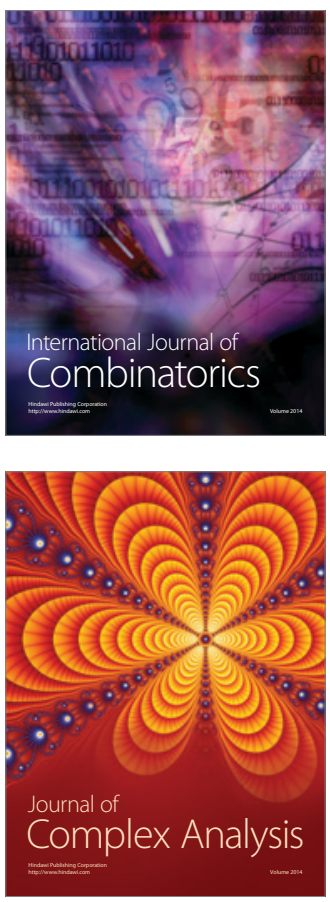

International Journal of

Mathematics and

Mathematical

Sciences
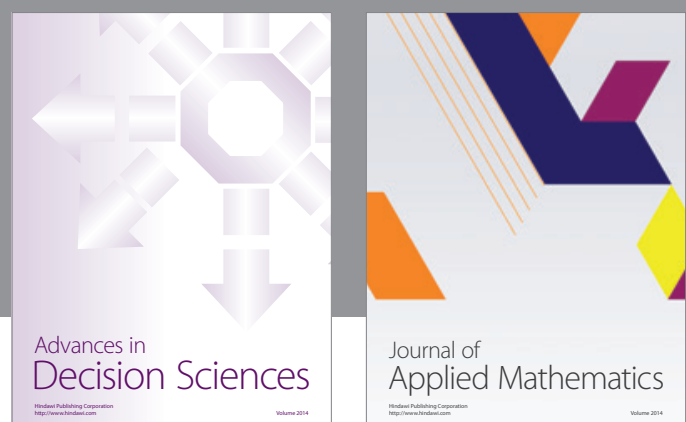

Journal of

Applied Mathematics
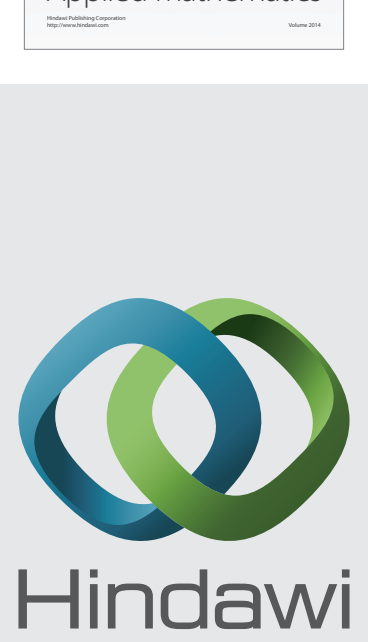

Submit your manuscripts at http://www.hindawi.com
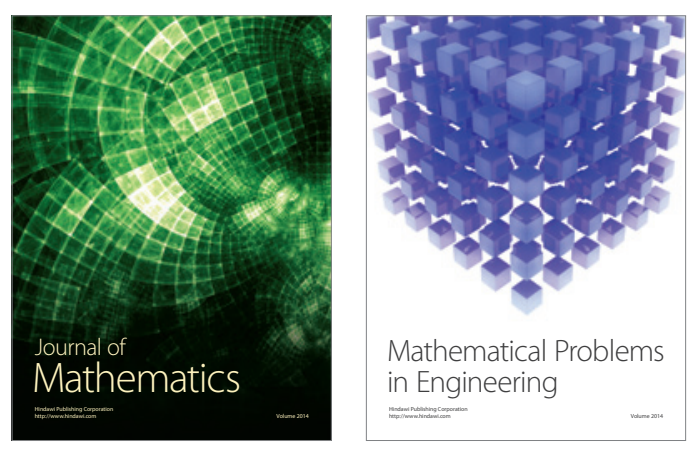

Mathematical Problems in Engineering
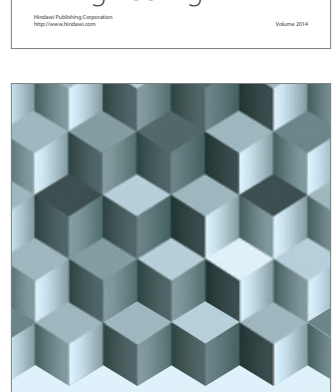

Journal of

Function Spaces
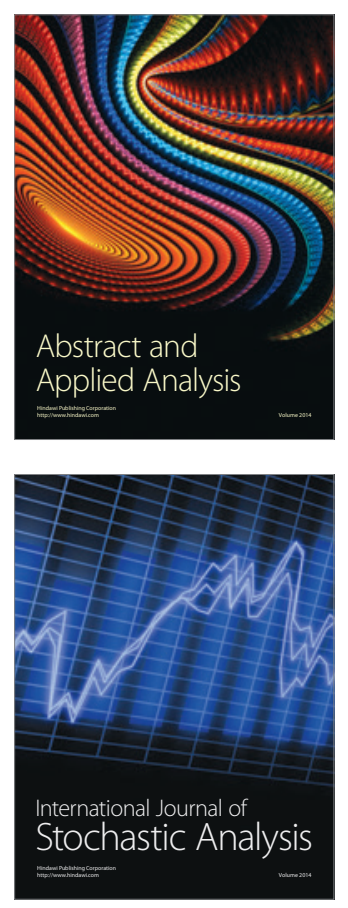

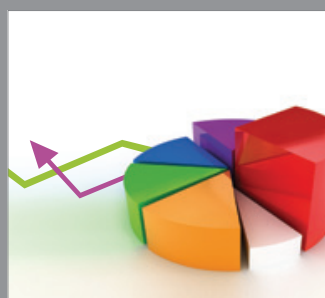

ournal of

Probability and Statistics

Promensencen
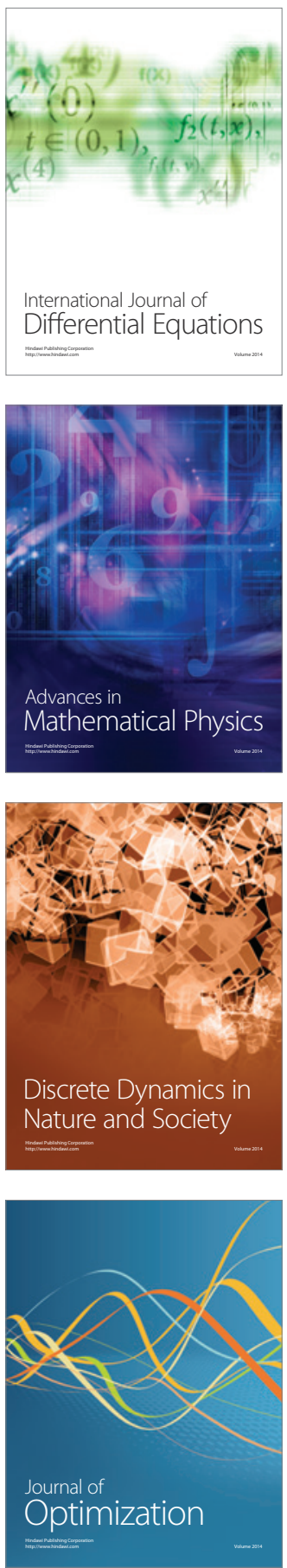